\title{
SISTEMA DE INFORMAÇÃO GEOGRÁFICA COMO APOIO AO LEVANTAMENTO DETALHADO DE SOLOS DO VALE DOS VINHEDOS $^{(1)}$
}

\author{
Eliana Casco Sarmento ${ }^{(2)}$, Carlos Alaberto Flores ${ }^{(3)}$, Eliseu Weber ${ }^{(4)}$, \\ Heinrich Hasenack ${ }^{(4)} \&$ Reinaldo Oscar Pötter ${ }^{(5)}$
}

RESUMO

O presente trabalho propõe o emprego de geotécnicas no apoio a um levantamento detalhado de solos, desde a coleta de dados em campo até à delimitação das unidades de solo e, posteriormente, a elaboração de um mapa final. A área de estudo corresponde a uma carta topográfica na escala 1:5.000 do Vale dos Vinhedos, na região da Serra Gaúcha, Rio Grande do Sul, Brasil. O material utilizado consiste em dados planialtimétricos de um levantamento aerofotogramétrico, receptores GPS (Global Posigioning System) e softwares de SIG (Sistemas de Informação Geográfica). Os softwares de SIG foram utilizados para integrar os dados de campo com as informações cartográficas e para analisar o relevo por meio de um MNT (Modelo Numérico do Terreno). Os resultados mostraram que o método permite a obtenção de um mapa digital georreferenciado, em que as unidades de mapeamento estão fortemente associadas com as fases de relevo, melhorando sua consistência e confiabilidade e facilitando seu uso futuro para outras aplicações.

Termos de indexação: Zoneamento Vitivinícola, Pedologia, Modelo Numérico do Terreno.

\footnotetext{
(1) Trabalho financiado com recursos da FINEP e CNPq. Trabalho apresentado no XXXI Congresso Brasileiro de Ciência do Solo, 2007 (Gramado, RS).

(2) Bolsista CNPq. Universidade Federal do Rio Grande do Sul. UFRGS, Centro de Ecologia. Av. Bento Gonçalves, 9.500. C.P. 15007, CEP 91.501-970. Porto Alegre - RS, Brasil. e-mail: eliana.sarmento@ufrgs.br

(3) Pesquisador da Embrapa Clima Temperado, BR 392 Km 78, C.P. 403, CEP 96001-970, Pelotas - RS, Brasil. e-mail: flores@cpact.embrapa.br

(4) Professor da UFRGS, Centro de Ecologia. e-mail: eweber@portoweb.com.br; hhasenack@ufrgs.br

(5) Consultor Embrapa Uva e Vinho, Rua Livramento 555, C.P. 319, CEP 83411-000, Bento Gonçalves - RS, Brasil.
} 


\title{
SUMMARY: USE OF A GEOGRAPHIC INFORMATION SYSTEM FOR A DETAILED SOIL SURVEY OF THE VALE DOS VINHEDOS, RS, BRAZIL
}

\begin{abstract}
This study proposes the use of geotechnologies to support detailed soil surveys, from field surveys to the delimitation of mapping units and later the drawing of the final map. The study area corresponds to a topographic map on a scale of 1:5,000 of the Vale dos Vinhedos, in the Serra Gaúcha region, State of Rio Grande do Sul, Brazil. The material used consists of planialtimetric data derived from aerial photographs, navigation GPS (Global Positioning System), and GIS (Geographical Information System) software. The GIS program was used to integrate field data with cartographic information and with a DTM (Digital Terrain Model). Results show that the methodology can generate a georreferenced digital map in which soil mapping units are strongly associated with relief phases, improving the consistency and reliability as well as making the use in further applications easier.
\end{abstract}

Index terms: Viticultural Zoning, Pedology, Digital Elevation Model.

\section{INTRODUÇÃO}

A produção vitivinícola mundial tem apresentado significativas mudanças nos últimos anos com a consolidação de novas áreas produtoras em diversos países, vários dos quais com atividades recente. No Brasil, houve, nas últimas décadas, significativo aumento da quantidade e da qualidade da produção vitivinícola interna, com a expansão de regiões produtoras e com o desenvolvimento de novos pólos de produção. Atualmente, cerca de $40 \%$ dos vinhos finos consumidos no País são nacionais, respondendo o Estado do Rio Grande do Sul por 95 \% da produção, com uma área de cerca de cinco mil hectares plantados com uvas viníferas.

A principal região produtora é a Serra Gaúcha, localizada no nordeste do Estado. As condições naturais da região são heterogêneas, com relevo complexo (altitude, declividade e exposição) e grande variabilidade de solos (Sarmento et al., 2006). Com origem vinculada à imigração italiana, a viticultura é uma atividade de pequenas propriedades rurais, com média de 15 ha de área total e 2,5 ha de vinhedos, topografia acidentada, pouca mecanização e predomínio da mão-de-obra familiar (Protas et al., 2004).

Dadas estas características, em um cenário de forte competição nos mercados (interno e externo), torna-se necessário um grande esforço de organização e de política setorial do Estado do Rio Grande do Sul, orientado para a busca de produtos distintos em características e tipicidade a fim de garantir a lucratividade e a manutenção da atividade vitivinícola da região.

A primeira iniciativa neste sentido foi a implementação de uma Indicação Geográfica, no caso, a Indicação de Procedência Vale dos Vinhedos (Tonietto \& Mandelli, 2005). Em que pese o pioneirismo deste trabalho, as informações disponíveis e utilizadas para caracterizar a região mostraram pouco detalhamento, impossibilitando a caracterização em um nível suficientemente específico para a implementação de uma Denominação de Origem.

Sabe-se que a qualidade da uva é o resultado da interação de vários fatores, como o clima, o sítio ou topografia local, o solo e a geologia, o cultivar escolhido e as práticas de manejo adotadas na produção (Vaudour, 2002; Jones et al., 2004), muito embora seja difícil definir a contribuição exata de cada um destes fatores, considerando a complexidade de suas interrelações (Wilson, 1998 citado por Jones et al., 2004). De todos os fatores ambientais, é o clima que exerce efeito determinante sobre a produção de uvas de qualidade em determinada região; todavia, na escala local, outros aspectos físicos assumem grande importância como, por exemplo, o solo. Por tal razão, ações ou políticas que busquem orientar a atividade no sentido da obtenção de produtos distintos em características e tipicidade devem necessariamente contemplar levantamentos de solos.

Os levantamentos de solos são realizados de acordo com métodos específicos que objetivam a identificação, caracterização e classificação das unidades taxonômicas ocorrentes em um sistema de classificação vigente, bem como sua delimitação para a obtenção de produtos finais na forma de cartas ou mapas. O processo contempla um estudo do terreno e das principais características dos perfis de solo, compreendendo descrição morfológica, caracterização física e química, classificação taxonômica e espacialização dos limites das unidades cartográficas. Quanto mais detalhado for o levantamento, mais homogêneas serão as unidades de mapeamento delimitadas (Flores et al., 2006).

As relações entre os solos e as formas da paisagem têm sido por longo tempo a base do mapeamento de 
solos. Tradicionalmente, a delimitação das unidades de mapeamento é feita por meio da análise estereoscópica de fotografias aéreas para o delineamento manual das unidades de solo-paisagem, que, posteriormente, serão identificadas e caracterizadas no campo (Ippolit et al., 2003).

Atualmente, a disponibilidade de novas técnicas GPS (Global Positioning System), PDA (Personal Digital Assistants) e SIG (Sistemas de Informação Geográfica) - abre novas possibilidades para o aperfeiçoamento dos mapeamentos de solos. Com efeito, elas podem ser utilizadas no apoio às diferentes fases do levantamento, contribuindo para acelerar e objetivar a coleta de dados em campo, melhorar a exatidão na delimitação das unidades de mapeamento, facilitar a disponibilização do produto final e potencializar os usos e aplicações dos resultados (Aronoff, 1991; Morris et al, 2000; Hempel et al., 2006).

O presente trabalho propõe a utilização de dados digitais de levantamentos aerofotogramétricos para a delimitação das unidades de mapeamento de solos, com base nas ferramentas de SIG. O objetivo foi avaliar a separação quantitativa das fases de relevo por meio da análise de um MNT, com vistas em auxiliar na individualização dos polígonos de solos no levantamento detalhado do Vale dos Vinhedos, Estado do Rio Grande do Sul, Brasil.

\section{MATERIAL E MÉTODOS}

A área de estudo situa-se no Vale dos Vinhedos, na região da Serra Gaúcha, na porção nordeste do Estado do Rio Grande do Sul. Compreende uma superfície de 673,5 ha, correspondente ao retângulo de uma carta na escala 1:5.000 da articulação que constituirá o levantamento detalhado de solos do Vale dos Vinhedos (Figura 1). O estudo foi desenvolvido em apenas uma carta (2952/2-s-2) com o objetivo de desenvolver e testar métodos que possam ser aplicados nas demais cartas do levantamento, visando a um produto final de maior consistência e qualidade.

O material utilizado para o desenvolvimento do trabalho consistiu em receptores GPS de navegação, software de edição vetorial Cartalinx (Clarklabs@), softwares de SIG Idrisi (Clarklabs() e Arcview (ESRIC), e uma base cartográfica digital da área de estudo. A base cartográfica foi produzida por empresa especializada por meio de um levantamento aerofotogramétrico na escala 1:10.000 (ampliadas para a escala 1:5.000 como material de trabalho de campo), com restituição digital da planimetria e da altimetria (curvas de nível com equidistância de $5 \mathrm{~m}$ ) e elaboração de um mosaico digital das fotografias ortorretificadas.

O primeiro passo foi estruturar a base cartográfica digital em ambiente de SIG, de forma a possibilitar a realização das análises necessárias e a geração das informações de interesse. Esta etapa envolveu a seleção e extração dos layers (camadas) úteis de cada folha do aerolevantamento, que se encontravam em formato de software CAD (Computer Aided Design), sua concatenação e a edição das bordas das folhas para a união dos elementos entre folhas adjacentes. Como resultado, obteve-se um conjunto de planos de informação individuais e contínuos, topologicamente estruturados e vinculados a tabelas com os atributos dos elementos.

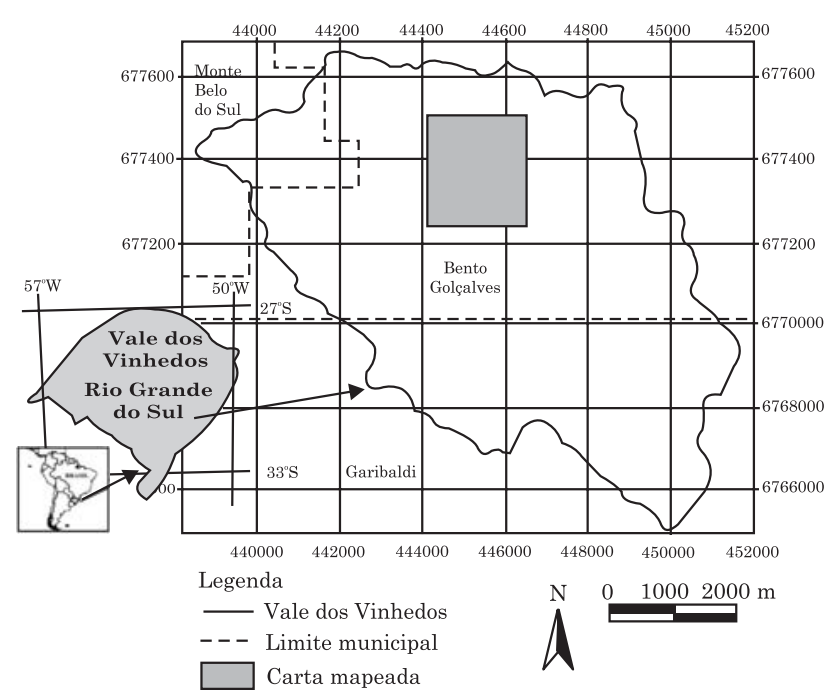

Figura 1. Localização da área de estudo.

Em seguida, a partir do plano de informação com as curvas de nível, foi gerado um Modelo Numérico do Terreno (MNT) pela interpolação linear baseada em uma rede triangular irregular (TIN). Para melhorar a qualidade do resultado, empregou-se uma função parabólica para ajustar as quebras de relevo e eliminar os efeitos de "ponte" e de "túnel" (estimar a altitude do fundo dos vales e do topo de elevações). A opção pela interpolação TIN levou em consideração as vantagens que esse tipo de modelo introduz na representação de áreas com relevo complexo (Câmara \& Medeiros, 1996). Para facilitar o uso do MNT, os valores de elevação derivados do TIN foram interpolados e convertidos no formato raster (matricial), utilizando uma resolução espacial de $5 \mathrm{~m}$ (célula de 5 × $5 \mathrm{~m}$ ).

A partir do MNT, efetuou-se o cálculo da declividade, que representa a primeira derivada da altitude e corresponde à inclinação da superfície do terreno em relação ao plano horizontal. Como resultado, obteve-se um novo plano de informação raster em que cada célula armazena o valor de sua inclinação. A declividade evidencia os processos de pedogênese e apresenta forte associação com os tipos de solo, especialmente em áreas com relevo complexo. Dessa forma, objetivando delimitar as distintas 
unidades de paisagem, utilizaram-se as classes de relevo usualmente utilizadas nos levantamentos de solos e modificadas por Flores et al (2006), definidas em sete classes de declividade, a saber: relevo plano (0-3 \%), suave ondulado (3-8 \%), moderadamente ondulado (8-13\%), ondulado (13-20\%), forte ondulado (20-45\%), montanhoso (45-75\%) e escarpado (>75\%). Esses intervalos foram empregados para efetuar o fatiamento do mapa de declividades anteriormente calculado e gerar um novo mapa com a delimitação das classes de declividade.

O mapa de classes de declividade e o mosaico de fotografias ortorretificadas foram utilizados como base para a elaboração de cartas de campo, criando-se um layout onde foram adicionadas as curvas de nível, uma grade de coordenadas UTM, a localização da folha na articulação do levantamento, a indicação de Norte, escala e outros elementos acessórios. As cartas de campo foram impressas e plastificadas com filme fosco para conferir resistência contra o manuseio e intempéries, bem como para facilitar o registro de informações visualizadas em campo sobre elas.

O trabalho de campo seguiu o método usual dos levantamentos de solos, em consonância com o Sistema Brasileiro de Levantamento e Classificação de Solos (Embrapa, 2006). Foram utilizados receptores GPS para o registro das coordenadas dos pontos de descrição de perfis de solo, de observação e de coleta de amostras para análise. O uso do GPS e de cartas de campo com grade de coordenadas permitiu a localização exata e instantânea de cada ponto sobre a carta em qualquer momento do trabalho de campo, facilitando o planejamento dos percursos a serem realizados e a definição de novos locais a serem visitados. Em conseqüência, à medida que se acumulava conhecimento sobre as relações solo-paisagem da área estudada, o trabalho de campo pôde ser constantemente adequado e otimizado, evitando coletas desnecessárias e possibilitando equilibrar a densidade de amostragem para as diferentes classes de solo. Durante esta etapa, as unidades de solos foram delineadas manualmente sobre as cartas de campo plastificadas, utilizando-se lápis dermatográfico (Figura 2).

Após a conclusão dos trabalhos de campo, a carta de campo plastificada, sobre a qual foram delimitadas as unidades de mapeamento de solos, foi digitalizada em scanner e georreferenciada com base na sua grade de coordenadas. Os limites das unidades de mapeamento foram então vetorizados manualmente em tela, utilizando-se a carta de campo georreferenciada como pano de fundo. O plano de informação vetorial resultante deste processo foi sobreposto ao mapa de classes de declividade (Figura 3) para efetuar os últimos ajustes nos limites de modo a melhorar a associação das unidades de mapeamento de solos às fases de relevo. A etapa final envolveu o fechamento dos limites e a construção dos polígonos, sua associação às unidades de mapeamento em uma tabela de atributos vinculada e a elaboração de um layout final do mapa de solos.

\section{RESULTADOS E DISCUSSÃO}

A figura 2 mostra a folha de campo com a delimitação das unidades de mapeamento feitas manualmente a partir das observações em campo com lápis dermatográfico sobre a carta plastificada. Observa-se, também, a localização de pontos visitados para descrição de perfis, observação dos solos e coleta de amostras, cujas coordenadas foram registradas com GPS e inseridas sobre a folha após seu escaneamento e georreferenciamento com o auxílio de SIG.

Analisando a figura 2, constata-se a excelente qualidade da folha de campo e a facilidade com que propicia a associação das classes de solos com a paisagem. O mosaico de fotografias aéreas ortorretificadas tem alto detalhamento, facilitando a correlação das observações de campo com seu aspecto na carta. As curvas de nível sobrepostas às fotografias também inserem valiosa informação sobre a topografia, possibilitando a identificação visual da posição de determinada área no relevo. Além disso, a grade de coordenadas fornece o referencial para o correto posicionamento do observador durante o trabalho de campo, uma vez que permite identificar o local exato de um ponto cujas coordenadas sejam registradas com o GPS. Estes aspectos facilitaram a delimitação das unidades de mapeamento, que aparecem com o traçado do lápis dermatográfico em vermelho, mas não permitiram reduzir a densidade de amostragem. A maior contribuição é a facilidade na delimitação das unidades de mapeamento e a otimização na distribuição dos pontos de amostragem nas unidades, possibilitando melhor caracterização, além da racionalização no planejamento e execução do trabalho de campo, reduzindo o esforço e o tempo necessários para sua conclusão.

A figura 3 mostra os limites das unidades de mapeamento depois de digitalizados, vetorizados e sobrepostos ao mapa de classes de declividade com o auxílio de SIG. Verifica-se um bom ajuste das unidades de mapeamento às fases de relevo, mesmo tratando-se de uma delimitação manual preliminar. Essa concordância, em grande parte, decorre da facilidade de associação das unidades de paisagem observadas (relevo) com os seus correspondentes na carta de campo, auxiliado pelo posicionamento com o GPS e pela experiência do pedólogo. A sobreposição com o mapa de classes de declividade possibilita, ainda, realizar um ajuste fino dos limites para produzir um mapa em que as unidades de mapeamento estejam altamente consistentes com as fases de relevo propostas, melhorando sua exatidão e confiabilidade. Em regiões com relevo mais suave outros elementos do aerolevantemento também podem ser utilizados para auxiliar neste ajuste, como os padrões de drenagem e de uso do solo, por exemplo. 


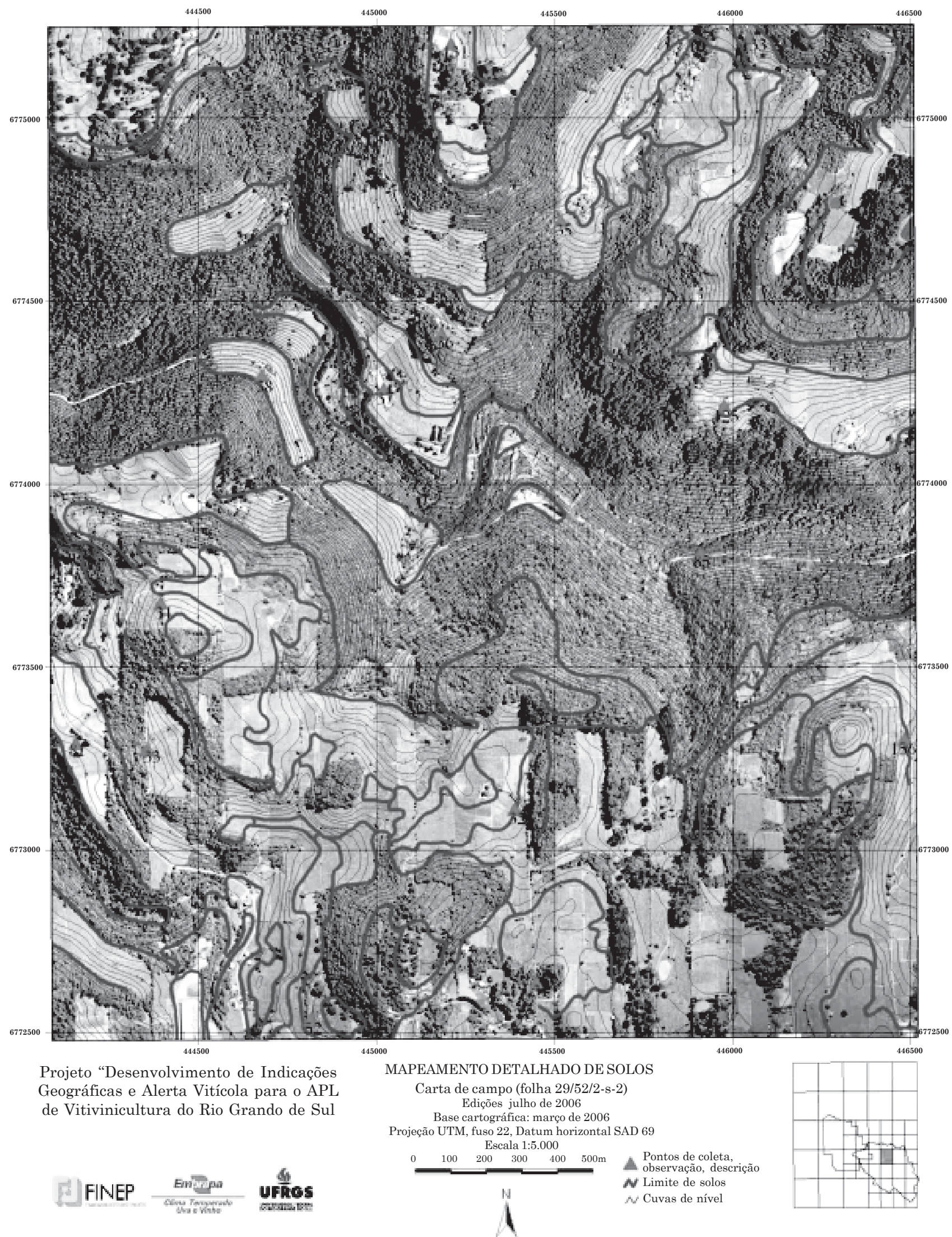

Figura 2. Carta de campo com o traçado manual dos limites das unidades de mapeamento. 


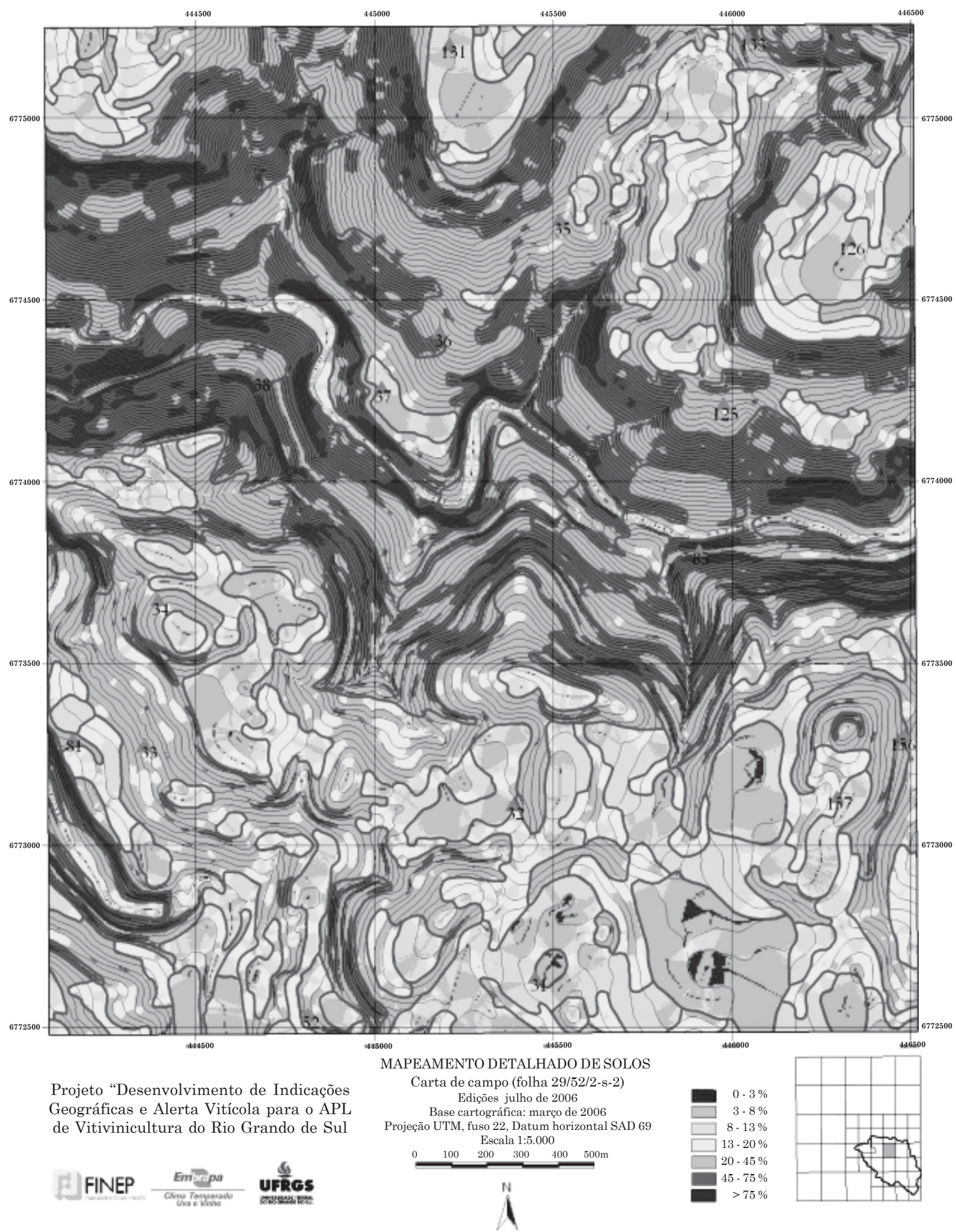

Figura 3. Limites das unidades de mapeamento vetorizadas sobrepostas ao mapa de classes de declividade. 
O levantamento detalhado de solos totalizou 37 unidades de mapeamento, sendo 10 da classe dos Argissolos, 16 da classe dos Cambissolos, quatro da classe dos Chernossolos, seis da classe dos Neossolos e um Tipo de Terreno. A figura 4 mostra o layout final do mapa de solos, classificado conforme Embrapa
(2006). As cores foram atribuídas a grupos de solos em nível de ordem, mantendo-se as unidades de mapeamento pertencentes a cada ordem com a mesma cor. A distinção é feita pela inserção da simbologia da unidade de mapeamento no interior de cada polígono.

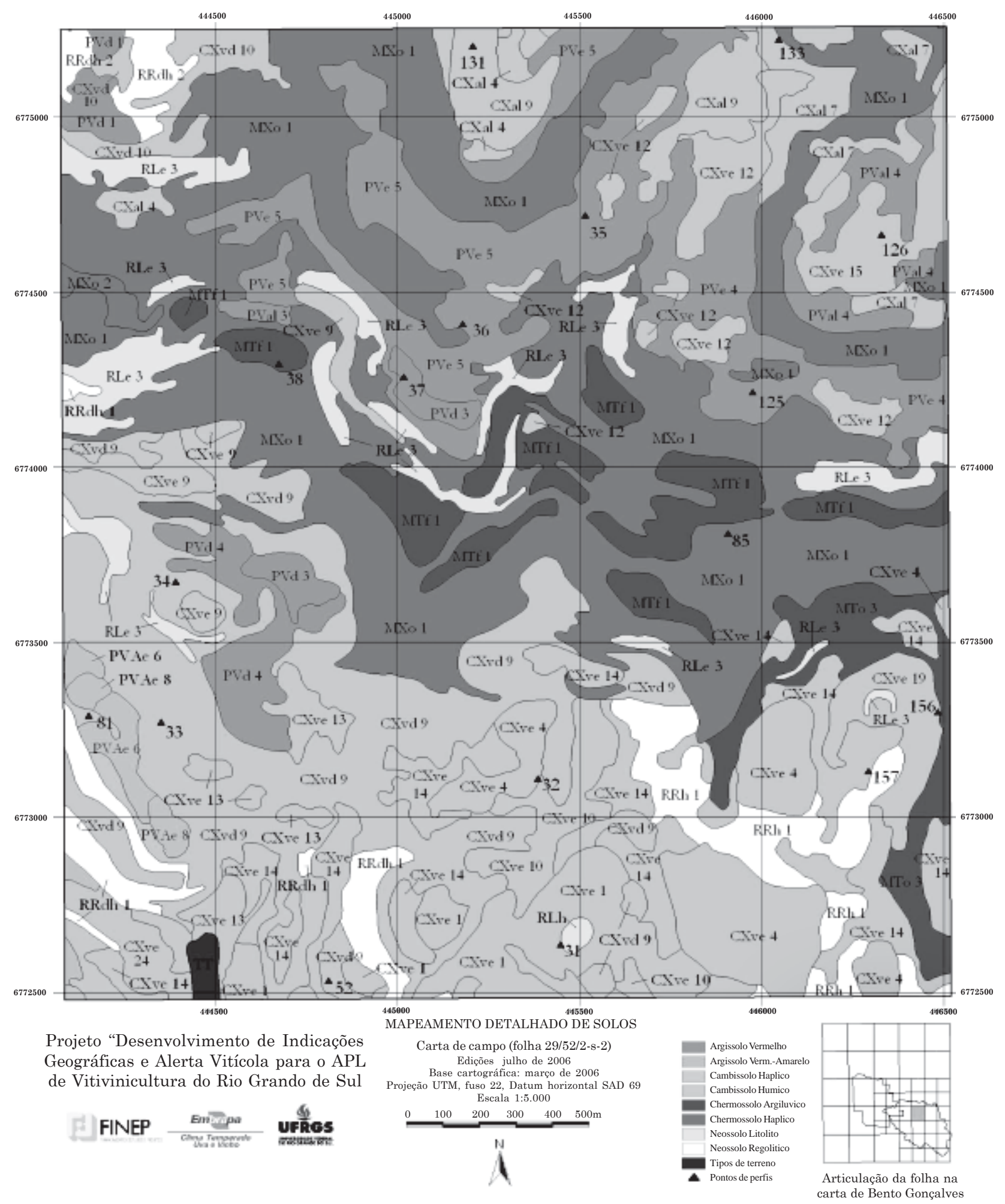

Figura 4. Mapa final de solos. 
A análise digital de um MNT para classificar as formas do relevo introduz vantagens em relação ao método tradicional para a separação das unidades de mapeamento: é uma alternativa rápida e bastante exata que pode ser aplicada para a quantificação e classificação do relevo, permitindo a definição automática ou semi-automática das unidades morfológicas da paisagem (Odeh et al., 1991; McBratney et al., 1991; Moore et al., 1991; Irvin et al., 1997).

O emprego de SIG nesse processo também torna possível efetivar análises quantitativas para verificar a adequação do mapa final de solos ao relevo, por meio do cálculo de índices de concordância como o Kappa, por exemplo. Para tanto, é necessário definir um conjunto de pontos amostrais a serem visitados para a obtenção da verdade de campo e o posterior cruzamento destes com o mapa. Esta atividade ainda não foi desenvolvida, mas como o produto final está em meio digital, georreferenciado e vinculado às principais informações descritivas e quantitativas das unidades de solos, sua execução não apresenta grandes dificuldades. Além disso, essas características também possibilitam a integração do mapa de solos com outros mapas e ampliam as possibilidades de uso para aplicações como zoneamentos, diagnósticos e avaliações de terras, dentre outros. Um mapa estruturado em SIG torna possível a realização de consultas sobre as características físicas, químicas ou biológicas dos solos de um local ou a seleção dos locais que apresentem solos com características de interesse. Também é possível quantificar as respectivas superfícies e cruzar as informações de solos com outros dados georreferenciados da região.

Levantamentos detalhados de solos sempre representam custos elevados, por isso sua produção deve ter em conta a maximização das suas utilidades e de seus benefícios. Mapas detalhados de solos são fundamentais para uma agricultura de exatidão ou, especificamente no caso do Vale dos Vinhedos, para uma viticultura de qualidade, que irá gerar produtos de alto valor agregado. Além de permitir melhor planejamento da atividade vitivinícola, o mapa detalhado de solos irá colaborar para a caracterização e aprimoramento da Indicação Geográfica do Vale dos Vinhedos quanto à uma futura Denominação de Origem.

\section{CONCLUSÕES}

1. O método testado mostrou-se adequado para a obtenção de mapas detalhados de solos em meio digital, georreferenciados e com maior consistência.

2. O uso de geotécnicas facilitou o mapeamento de solos para: geração de material para impressão com rapidez e uniformidade; apoio no planejamento das atividades de campo; georreferenciamento das informações de coleta em campo; espacialização e edição dos limites das unidades de solos; conferência e correções dos dados e manutenção de consistência espacial e de atributos do mapa digital resultante.

\section{AGRADECIMENTOS}

À Financiadora de Estudos e Projetos (Finep) e ao CNPq, pelo apoio financeiro.

\section{LITERATURA CITADA}

ARONOFF, S. Geographic information systems: A management perspective. Otawa, WDL Publications, 1991. 294 .

CÂMARA, G. \& MEDEIROS, J.S. Geoprocessamento para projetos ambientais. São José dos Campos, INPE, 1996. $186 \mathrm{p}$.

EMPRESA BRASILEIRA DE PESQUISA AGROPECUÁRIA EMBRAPA. Sistema brasileiro de classificação de solos. 2.ed. Rio de Janeiro, 2006. 306p.

FLORES, C.A.; PÖTTER, R.O.; FASOLO, P.J.; HASENACK, H. \& WEBER, E., orgs. Levantamento semidetalhado de solos: Região da campanha - Folha Palomas, Estado do Rio Grande do Sul. Porto Alegre, Universidade Federal do Rio Grande do Sul, 2006.

HEMPEL, J.W.; HAMMER, R.D.; MOORE, A.C.; BELL, J.C.; THOMPSON, J.A. \& GOLDEN, M.L. Challenges to digital soil mapping. In: GLOBAL WORKSHOP ON DIGITAL SOIL MAPPING, 2., Rio de Janeiro, 2006. Proceedings. Rio de Janeiro, 2006. CD-ROM.

IPPOLIT, G.; COSTA, L.M.; GAGGERO, M.R.; SCHAEFFER, C.E.; FERNANDEZ FILHO, E.I. Uso de geoprocessamento para o levantamento preliminar de solos de uma microbacia da Zona da Mata (MG). In: SIMPÓSIO BRASILEIRO DE SENSORIAMENTO REMOTO, 11., Belo Horizonte, 2003. Anais. Belo Horizonte, 2003. p.153160 .

IRVIN, B.J.; VENTURA, S.J. \& SLATER, B.K. Fuzzy and isodata classification of landform elements from digital terrain data in Pleasant Valley, Wisconsin. Geoderma, 77:137-154, 1997.

JONES, G.; SNEAD, N. \& NELSON, P. Geology and wine 8. Modeling viticultural landscapes: A GIS Analysis of the Terroir Potential in the Umpqua Valley of Oregon. Geoscience, 31:167-178, 2004.

McBRATNEY, A.B.; HART, G.A. \& McGARRY, D. The use of region partitioning to improve the representation of geostatiscally mapped soil attributes. J. Soil Sci., 3:513533,1991

MOORE, I.D.; GRAYSON, R.B. \& LADSON, A.R. Digital terrain modeling: A review of hydrological, geomorphological and biological applications. Hydrol. Proc., 5:3-30, 1991.

MORRIS, D.K.; STIENHARDT, G.C.; NIELSEN, R.L.; HOSTETTER, W.; HALEY, S. \& STRUBEN, G.R. Using GPS, GIS, and remote sensing as a soil mapping tool. In: INTERNATIONAL CONFERENCE ON PRECISION AGRICULTURE, 5., Bloomington, 2000. Procedings. St. Paul, Center for Precision Agriculture, University of Minnesota, 2000. (CD-ROM). 
ODEH, I.O.A.; CHITTLEBOROUGH, D.J. \& McBRATNEY, A.B. Elucidation of soil-landform interrelationships by canonical ordination analysis. Geoderma, 49:1-32, 1991.

PROTAS, J.F.S.; CAMARGO, U.A. \& MELLO, L.M.R. A vitivinicultura brasileira: Realidade e perspectivas. Embrapa Uva e Vinho. Disponível em: <http://www. embrapa.cnpuv.embrapa.br/publica/artigos/ vitivinicultura/>. Acesso em 31 ago. de 2004.

SARMENTO, E.C.; WEBER, E.; HASENACK, H.; TONIETTO, J. \& MANDELLI, F. Topographic modeling with GIS at Serra Gaúcha, Brazil: Elements to study viticultural terroir. In: TERROIRS VITICOLES, 6., Bordeaux, 2006. Anais. Bordeaux, ENITA, 2006. v.1. p 365-372.
TONIETTO, J. \& MANDELLI, F. Como organizar, promover y reconocer regiones de excelencia de producción de vinos: Una experiencia de Brasil en indicadores geográficos. In: SEMINARIO INTERNACIONAL DE VITIVINICULTURA, 2., Ensenada, 2005. Mexico, Inifap, 2005. p.7-19.

VAUDOUR, E. The quality of grapes and wine in relation to geography: Notions of terroir at various scales. J. Wine Res., 13:117-141, 2002.

WILSON, J.E. Terroir: The role of geology, climate, and culture in the making of french wines: Mitchell Beazley.London, 1998. 336p. 
